\title{
Intervención de las instituciones en la reducción de la fecundidad y la mortalidad infantil*
}

\author{
Fátima Juárez**
}

Históricamente las instituciones han actuado como mediadoras de las relaciones sociales. Ellas tienen características específicas, historia autónoma y evolución propia. Desde esta perspectiva teórica, cada una de las instituciones se podría considerar como la vinculación entre la conducta individual y la esfera macro (reproducción social, ideología, infraestructura, etc.). Algunas de ellas, como las instituciones de salud y educación, tienen una incidencia más directa en la dinámica demográfica de la familia.

Desde esta perspectiva, el presente trabajo examina las instituciones y algunas de sus intervenciones específicas, en relación con la influencia que éstas pueden tener en las estrategias individuales de la reproducción de la familia. Bajo este enfoque se considera que la conducta individual puede ser influida y caracterizada por su ámbito económico y social.

Este documento discute el impacto de las instituciones sociales en la experiencia de la reproducción de las fomilias y la reproducción de la sociedad. A pesar de las dificultades empíricas para demostrar la asociación entre las esferas micro y macro, el documento presenta evidencia de esta relación al introducir el caso de Ecuador. Para ello, se estiman índices globales de las estrategias reproductivas de las fomilias (fecundidad) y de su experiencia de sobrevivencia de los hijos (mortalidad infantil). Se utiliza la disponibilidad de servicios en el área de residencia como una aproximación del impacto potencial de estas instituciones, y se enlaza con la información de nivel micro sobre la experiencia individual de la reproducción y la muerte de los hijos. En el estudio se hace uso de los datos provenientes del cuestionario individual y de la comunidad pertenecientes al programa de la Encuesta Demográfica y de Salud (DHs).

Los resultados muestran que las instituciones de educación y de salud juegan un papel influyente en la dinámica del comportamiento demográfico de los individuos. Las intervenciones específicas de salud, como el programa salud materno-infantil o el de rehidratación oral, están ligadas a la reducción de la fecundidad y de la mortalidad infantil, respectivamente.

* El presente artículo es una versión modificada de la ponencia presentada en la Conferencia del Poblamiento de las Américas.

** Naciones Unidas, División de Población. Los puntos de vista y opiniones expresadas en este documento son de la autora y no reflejan necesariamente los de Naciones Unidas. 


\section{Introducción}

La investigación ha puesto de manifiesto el descenso de la fecundidad y la mortalidad infantil en la mayoría de los países de América Latina. Los índices demográficos clásicos y el análisis detallado del proceso de reproducción y de la sobrevivencia de los hijos han demostrado estos cambios. En la mayoría de los estudios empíricos, la explicación de la reducción en la fecundidad y la mortalidad infantil se basa en sus características y en las metas de los individuos, aunque se reconoce la importancia de que la conducta individual está influida y determinada por el ámbito socioeconómico en donde se desarrolla.

En la interpretación de la conducta demográfica han surgido diferentes marcos teóricos; el presente trabajo parte de la conceptualización de que las instituciones sociales actúan como mediadoras de los cambios en las actitudes hacia la conducta reproductiva y las respuestas a la sobrevivencia de los hijos.

A lo largo de la historia, las instituciones han actuado como mediadoras de las relaciones sociales, además han presentado características y evolución propia. Cada institución, a saber, la iglesia, la salud, la ley, etc., adquiere una posición específica como mediadora entre la conducta del individuo y la esfera macro, entre ellas sobresalen algunas porque están vinculadas más directamente a la estrategia de la reproducción humana (nacimiento y muerte), por decirlo así, son mediadoras de los cambios en el proceso de reproducción.

Un enfoque que incluya distintos niveles de análisis puede ayudar a la mejor comprensión de los cambios que están ocurriendo en la conducta reproductiva y en la mortalidad. Esta estrategia necesita información de nivel micro y macro. El "Cuestionario de la Comunidad" de la Encuesta Demográfica y de Salud Familiar de Ecuador (EDS) permite la posibilidad de estudiar el significado de los factores contextuales y su influencia en el comportamiento demográfico.

El propósito de este documento es presentar alguna evidencia empírica del vínculo de las instituciones y su intervención en los patrones de fecundidad y mortalidad infantil. El análisis se basa en la información de los cuestionarios tanto individual como de la comunidad de la EDS, realizada en Ecuador en $1987 .{ }^{1} \mathrm{El}$ énfa-

\footnotetext{
${ }^{1}$ La información de la EDS de Ecuador de 1987 (también conocida como Encuesta Demográfica y para la Salud Materna EDSM) fue coordinada por el Centro de Estudios y Paternidad Responsable (Cepar), y por el Instituto de Investigaciones Nutricionales y Médico Sociales (Ininms), bajo el Programa EDS IRD.
} 
sis se manifiesta en dos instituciones cuya influencia se puede medir a través de las encuestas de fecundidad: las instituciones de educación y de salud (en la definición de salud se incluyen tanto los servicios de salud como los de planificación familiar). La fecundidad y la mortalidad se estudian a través de índices de momento, además éstos se examinan conjuntamente de acuerdo con la disponibilidad o carencia de los servicios en la comunidad, asimismo se analizan las tendencias.

\section{La institución de salud: algunas consideraciones teóricas}

La transformación del comportamiento de los individuos y de la sociedad ha sido una cuestión fundamental entre los científicos sociales. Pero a pesar del interés que existe entre los sociólogos, economistas y demógrafos por entender la conducta del individuo y su relación con la esfera del macro nivel, han sido muy pocos los estudios que intentan investigar esta relación de una manera rigurosa (McNicoll, 1980, 1982; McNicoll y Cain, 1990; Casterline, 1985; Hermalin, 1986; Potter, Mojarro y Núñez, 1987; Entwisle, 1989; Tsui y Ochoa, 1989). Un nivel intermedio de la realidad, generalmente pasado por alto en el estudio de la reproducción humana y sus vínculos con la dimensión macro, es el de las instituciones sociales, que deben ser consideradas como la esfera ideológica con capacidad de actuar sobre la conducta reproductiva y la condición de sobrevivencia de la población. Así, las instituciones sociales se pueden concebir como entidades que establecen reglas y procedimientos para organizar la actividad práctica y legitimar las relaciones de clase. Con mayor o menor grado de éxito, ellas controlan y regulan su propia existencia, al tiempo que suministran bienes y servicios para la subsistencia de la sociedad en su conjunto.

La conceptualización teórica que se retoma en el presente estudio es la que considera a las instituciones sociales como mediaciones de las relaciones sociales. Ésta se basa en los trabajos de Loyola et $a$ l. (1982) y Camarena (1991), los cuales examinan la importancia de la institución de salud como mediadora del cambio.

Las instituciones son dominios sociales, culturales e ideológicos que regulan las relaciones de los individuos, grupos y familias. Desde una perspectiva global, no representan una entidad unificada ya que se pueden presentar contradicciones, las cuales llevan a que ciertas esferas se vuelvan más dominantes que otras. Las instituciones pueden tener una dimensión gubernamental, civil o religiosa que influye y actúa sobre múltiples dominios del contexto social. 
Entre las instituciones, las de salud, con sus intervenciones directas hacia la salud y la planificación familiar, son las que tienen la influencia directa más fuerte y significativa sobre el comportamiento reproductivo de la población y la condición de salud de los individuos. A causa de las modificaciones que han ocurrido en la sociedad, tales como el mejoramiento tecnológico y las acciones llevadas a cabo por las instituciones de salud, el control de la sexualidad y la reproducción (las muertes y los nacimientos) de la población se ven, cada vez más, como parte de la responsabilidad de estas instituciones.

Los efectos reales que la institución salud puede tener en la sobrevivencia de la población y en su reproducción están determinados por la compleja organización y la operacionalización de las instituciones, los múltiples agentes que intervienen en sus operaciones y las características socioeconómicas y culturales de los individuos hacia los que se dirigen las acciones. Entre los elementos que están involucrados en el proceso de mediación se cuentan los siguientes:

a) Los elementos subjetivos orientados a informar, persuadir y a crear conciencia sobre las ventajas de las medidas primarias de la salud y el espaciamiento de los nacimientos (a través de medios de comunicación masivos u otras formas).

b) La heterogeneidad dentro de la institución introducida por los múltiples agentes involucrados y por las circunstancias contextuales en particular. La transmisión y la interpretación de las normas y las acciones se ven afectadas por los intereses y los motivos de cada uno de los agentes que prestan los servicios de salud, los cuales dependen de sus valores y conocimientos acerca de la reproducción y de la familia. Por consiguiente, los agentes médicos son un factor clave como mediadores entre las disposiciones y las metas de las instituciones.

c) El grado de infraestructura.

d) El acceso y los costos.

e) La potencia de los programas.

f) La calidad del servicio.

g) La influencia de otros factores que pertenecen a la esfera socioeconómica y cultural, los cuales pueden facilitar u obstaculizar las metas.

La reducción de la mortalidad y la fecundidad no puede ser atribuida exclusivamente a la acción de las instituciones de salud y al efecto de la interacción del resto de las instituciones sociales, pues tienen objetivos que no van necesariamente en la misma di- 
rección. Un elémento adicional importante que contribuye a la modificación de estos eventos demográficos es el desarrollo y la aplicación de la medicina moderna.

Una manera de observar la expansión y alcance de la influencia de las instituciones de salud es mediante el examen de la evolución de los servicios que se ofrecen y su impacto en los niveles y las tendencias de la fecundidad y la mortalidad infantil. La creación de instalaciones médicas puede generar que dicha comunidad se convierta en un área de influencia para el resto de la sociedad. Las acciones concretas y especificas de la instauración de los servicios podrían servir para propósitos prácticos de análisis, como una aproximación para medir la influencia potencial de la institución salud.

La existencia de servicios puede ser utilizada como una medida de las intervenciones de las instituciones de salud. Esta variable próxima se puede mejorar si se incluye la condición de accesibilidad. Las estimaciones no pueden proveer una descripción del proceso completo y su dimensión (los mecanismos de interacción, las relaciones de conflicto y la diversidad), pero sí representan las consecuencias demográficas de las intervenciones institucionales, es decir, la modificación que se pueda dar en la conducta reproductiva y en la sobrevivencia de los hijos.

Otros agentes, como el farmacéutico, la partera y el médico tradicional, que no están formalmente integrados dentro de los servicios gubernamentales de salud, se pueden considerar como agentes complementarios de la institución de salud. Por ejemplo, aunque los establecimientos farmacéuticos tienen un radio de acción limitado, pueden jugar un papel muy importante de apoyo o influencia en las acciones relacionadas con los aspectos de salud. Como en la mayoría de los países de América Latina, en Ecuador, la función del farmacéutico ha sido extremadamente importante. En general, ellos sustituyen a los médicos en el diagnóstico, las prescripciones e instrucciones del uso de los medicamentos y los métodos anticonceptivos. En muchos casos, la existencia de estos establecimientos, junto con otros agentes de salud no formales como las parteras y los doctores tradicionales, contribuyen a la difusión de la salud y de la planificación familiar.

Información, metodología y definiciones: el caso de Ecuador

Fuente de datos y metodología

Los datos utilizados provienen de la EDS que pertenece al programa de la Encuesta Demográfica y de Salud (DHS). La encuesta 
recolectó información individual y de la comunidad, ésta se obtuvo en el llamado módulo de disponibilidad y de servicios, en donde se recogieron datos sobre el nivel de la infraestructura actual y la disponibilidad de los servicios de salud y planificación familiar públicos y privados. En forma adicional, se captaron datos sobre la evolución histórica de la disponibilidad de los servicios específicos de salud. La información de la comunidad de la EDS representa, para Ecuador, la fuente más reciente de información de este tipo. ${ }^{2}$

Los datos del cuestionario que se utilizan en el análisis -archivo individual - son la historia de nacimientos y algunas características socio-demográficas de las mujeres en edad reproductiva (15-49 años). Del cuestionario sobre disponibilidad de servicios -archivo de la comunidad- se aprovecha la información recabada sobre la existencia de los establecimientos de salud y planificación familiar, y la fecha de iniciación de dichos servicios. A los servicios específicos que otorgan los establecimientos se les denomina programas para distinguirlos de las facilidades de servicios de salud. Los programas que se consideran en el análisis son: el relàcionado con el cuidado de la salud materno-infantil, el de planificación familiar y el de rehidratación oral.

En relación con el archivo de la comunidad, hay que resaltar que no solamente para el caso de la EDS de Ecuador, sino también para todas las encuestas pertenecientes al Programa de Encuestas Demográficas y de Salud y de la Encuesta Mundial de Fecundidad, la información del cuestionario se ha utilizado muy poco y no se han analizado con profundidad las deficiencias que existen en la formulación de las preguntas y la captación de los datos recabados. Por esta razón, fue necesario evaluar y analizar la consistencia de cada una de las preguntas del cuestionario; después de una exploración profunda, se encontró que solamente un número reducido de variables eran significativas para el análisis. Otra limitación del cuestionario se refiere al concepto "disponibilidad del establecimiento" en la comunidad, éste alude al conglomerado utilizado para la muestra y no a un concepto de "comunidad" o a una división geográfica o a otra área socialmente reconocida, lo cual hubiera sido más apropiado. En el análisis, la disponibilidad de los servicios se utiliza como un sustituto para conocer la influencia que tiene la institución salud sobre la sobrevivencia infantil y la conducta reproductiva.

\footnotetext{
${ }^{2}$ No se recolectó ninguna información directa del establecimiento de delegaciones médicas.
} 
La muestra del cuestionario individual de la EDS en Ecuador tiene representación regional. El módulo de disponibilidad de servicio se recolectó para 296 segmentos (conglomerados que corresponden a sectores censales), 120 urbanos y 76 rurales.

La información de la comunidad se relaciona con la proveniente de la historia de nacimientos, de esta manera obtenemos como resultado un cuadro sobre el efecto de la evolución de la disponibilidad del servicio específico de la salud durante los últimos 35 años en relación con la historia de nacimientos. Ésta es la información básica que se util za para la obtención de los indicadores.

La Tasa Global de Fecundidad (TGF) y la Tasa de Mortalidad Infantil (TMI) son los dos índices clásicos que se calculan en este trabajo. La historia de nacimientos cuenta con información sobre el acontecimiento mes a mes, por lo que esta información detallada se aprovecha para los cálculos, y se estima mensualmente la exposición al riesgo. Con los indicadores así calculados se realizan dos tipos de análisis: uno de momento y otro sobre las tendencias.

En el análisis de momento, las tasas se refieren a los últimos 5 años anteriores a la fecha de la encuesta y se asocian con la información actual sobre la existencia de servicios médicos y educativos. Con respecto al análisis de las tendencias, los estimadores que se utilizaron en la presentación de la evolución de las tasas de mortalidad infantil y la fecundidad se calcularon considerando la disponibilidad de los centros de salud y de los programas al tiempo de la ocurrencia del evento, nacimiento o muerte del hijo. El periodo de referencia para las TGF es de cinco años, mientras que, debido a los problemas del tamaño de la muestra, las TMI se agruparon en periodos de 10 años. Dado que la información es de tipo retrospectivo, al retroceder en el tiempo, la experiencia de las mujeres está truncada. Para eliminar el sesgo que introducen las diferentes exposiciones al riesgo, se utilizaron estimadores para edades comparables, de manera que para las estimaciones de la fecundidad, solamente se consideraron, para cada periodo, a las mujeres que tenían de 15 a 35 años de edad.

\section{Estructura de los servicios}

El análisis de nivel macro no se puede entender o examinar si no se consideran las especificidades de la sociedad. Por esta razón, a continuación se presentará alguna información relevante sobre los conceptos administrativos del país y los conceptos del cuestionario.

La división política administrativa de Ecuador sigue un orden 
descendente: provincias, cantones y parroquias. Las áreas principales de las provincias y de los cantones se consideran urbanas y se denominan cabeceras. ${ }^{3} \mathrm{Al}$ momento de la encuesta (1987) había 21 provincias, 147 cantones y 712 parroquias. ${ }^{4}$

Existen tres niveles educativos en el país: primaria, secundaria (colegio y colegio técnico) y universidad. Las escuelas están distribuidas en todo el país y las universidades sólo se encuentran en las áreas urbanas.

Respecto a la prestación de servicios de salud, hay una estructura que está regulada por el Ministerio de Salud Pública. En las áreas rurales solamente existen centros y subcentros de salud, además de otras unidades más pequeñas como son los puestos de salud y dispensarios que no cuentan con un médico. ${ }^{5}$

En las áreas urbanas, la infraestructura que provee el gobierno está formada por centros de salud, hospitales generales y especializados, estos últimos se localizan regularmente en las cabeceras provinciales. Debido a las estructuras administrativas específicas, cada servicio de salud atiende a diferentes subgrupos de población. En particular, llama la atención que las facilidades de los hospitales se localizan sólo en áreas urbanas y que la población rural en caso de necesitar el servicio tiene que ser canalizada a los hospitales urbanos.

En comparación con otros países de la región (como México), en el Ecuador, el gobierno no ha reforzado directamente las actividades de planifícación familiar. Los hospitales y los centros de salud pueden suministrar servicios de planificación familiar, pero en sentido estricto no los promueven. El gobierno no es el único agente responsable del diseño, administración y puesta en práctica de los programas de planificación familiar. Esta tarea la comparte con el sector privado, por ejemplo, las clínicas privadas, los médicos particulares y las agencias no gubernamentales tales como CEMOPLAF y APROFE, ${ }^{6}$ las cuales han demostrado su com-

3 Área principal del cantón.

4 El concepto de "periferia" fue creado específicamente para el censo (concepto operacional) y se le trató igual que a las zonas urbanas. Le corresponde el área alrededor de la cabecera del cantón, sin embargo, también puede pertenecer a una o más parroquias del mismo cantón.

5 El dispensario es una unidad elemental que sólo cuenta con servicios de primeros auxilios; este servicio está a cargo de promotores de salud y voluntarios. Por su parte los puestos de salud ofrecen exclusivamente el servicio de primeros auxilios, que están a cargo de una enfermera y fueron diseñados para cubrir 2000 habitantes.

${ }^{6}$ Centros médicos de orientación y planeación familiar y Asociación ProBienestar de la familia ecuatoriana. 
promiso en esta área. Los servicios de las agencias no gubernamentales, en la información de la EDS de Ecuador, se captaron en la categoría de clínicas, junto con los servicios privados.

La prestación de los servicios relacionados con el cuidado de la salud de la población, y en particular con la sobrevivencia de los hijos, ha estado principalmente bajo el control del gobierno.

Otro de los agentes del servicio de salud, de gran importancia en Ecuador, es el farmacéutico (personas que no tienen entrenamiento especializado). Su función es distinta a la que se observa en los paises desarrollados. El farmacéutico provee las medicinas al mismo tiempo que indica su modo de empleo. En muchos casos la sociedad los reconoce como proveedores de los medicamentos apropiados, aunque de tipo curativo en la mayoría de los casos. De la misma forma, ellos dan información sobre los anticonceptivos. Este servicio se podría considerar como más accesible para la población.

En el módulo de disponibilidad de servicios (cuestionario de la comunidad) de la EDS de Ecuador, se recolectó información sobre cinco tipos de establecimientos de salud: hospitales, centros de salud, clínicas, médicos particulares y farmacias. El gobierno brinda los servicios hospitalarios y de los centros de salud, y el resto son de carácter privado. Dada la estructura oficial de los servicios, los hospitales sólo se localizan en las áreas urbanas, mientras que los centros de salud se pueden encontrar indistintamente en áreas urbanas y rurales. Los centros de salud constituyen el servicio básico de salud a nivel primario más importante que el gobierno provee en las áreas rurales.

\section{Definición de disponibilidad y acceso}

A fin de encontrar una aproximación satisfactoria de la medición de las condiciones de la distribución de los establecimientos médicos, según la localidad de ubicación, fue necesario conocer las condiciones particulares del país y hacer un examen profundo de la comunidad. ${ }^{7}$ Se concluyó que los servicios estaban disponibles y eran accesibles si el establecimiento estaba dentro de un radio de dos kilómetros del lugar de residencia. En el análisis se utiliza-

${ }^{7}$ La tarea involucró no sólo el análisis de cada variable, sino que también la revisión de la localización en el mapa de los segmentos. Se requirió de la colaboración de un experto del país para ofrecer información detallada acerca de la nación y la disponibilidad de servicios. Quisiera agradecer a Marco Posso por contribuir en la etapa exploratoria y por su ayuda en el cálculo del IMR. 
rá siempre la definición disponibilidad-accesibilidad dentro de un radio de dos kilómetros.

Esta conceptualización de accesibilidad nos acerca al posible impacto que las acciones del gobierno pueden tener sobre la conducta de la fecundidad y la sobrevivencia de los hijos, y elimina en parte las dificultades que el individuo podría tener para utilizar estos servicios, al menos en el caso de los servicios públicos. Desafortunadamente, no se recolectó información sobre la calidad y cantidad de los servicios, lo cual hubiera permitido la construcción de un indicador más refinado de cobertura y acceso.

Con el fin de ilustrar las limitaciones de la definición original de existencia de servicio cercano al conglomerado, y del mejoramiento del nuevo concepto de disponibilidad-accesibilidad, en el cuadro 1 se presenta la distribución de ambas mediciones. Entre las comunidades muestreadas $100 \%$ registran la existencia de escuela primaria, $92 \%$ la de escuela secundaria y $50 \%$ la de universidades. Aunque es verdad que hay disponibilidad de escuelas primarias casi en todas partes del país, las universidades, tanto privadas como públicas, están situadas sólo en las ciudades grandes y los porcentajes que se registran bajo esta definición no reflejan la realidad.

Respecto a los servicios de salud se presenta la misma aparente sobrerrepresentación. Más de dos tercios de las áreas del país tienen hospitales, casi $\mathbf{8 0} \%$ de las comunidades tienen centros de salud (servicios bajo el Ministerio de Salud Pública) y un elevado porcentaje de comunidades tiene acceso al resto de los servicios.

El ejemplo más ilustrativo es la distribución de los servicios en el contexto rural. Cuando la disponibilidad se define como la existencia de servicios en el segmento, los datos muestran que $6.6 \%$ de los conglomerados rurales tienen universidades y $25 \%$ hospitales. Sin embargo, a la fecha de la encuesta, no habia universidades o colegios disponibles en las áreas rurales de Ecuador y, como se comentó anteriormente, por definición de la estructura de salud del gobierno no existen hospitales en las áreas rurales. Esto muestra claramente las deficiencias de la definición utilizada por la encuesta, en relación con la existencia y disponibilidad de servicios en los conglomerados.

Con el nuevo concepto de disponibilidad-accesibilidad (en un radio de dos kilómetros) se muestra que $1.3 \%$ de los conglomerados rurales tienen acceso a universidades y $3.9 \%$ a hospitales. Cuando se verifica esto con el mapa, ${ }^{8}$ se ve que los puntos corres-

\footnotetext{
${ }^{8}$ La disponibilidad de hospitales en $3.9 \%$ corresponde al modelo de seis puntos rurales.
} 
REDUCCIÓN DE LA FECUNDIDAD Y LA MORTALIDAD INFANTIL

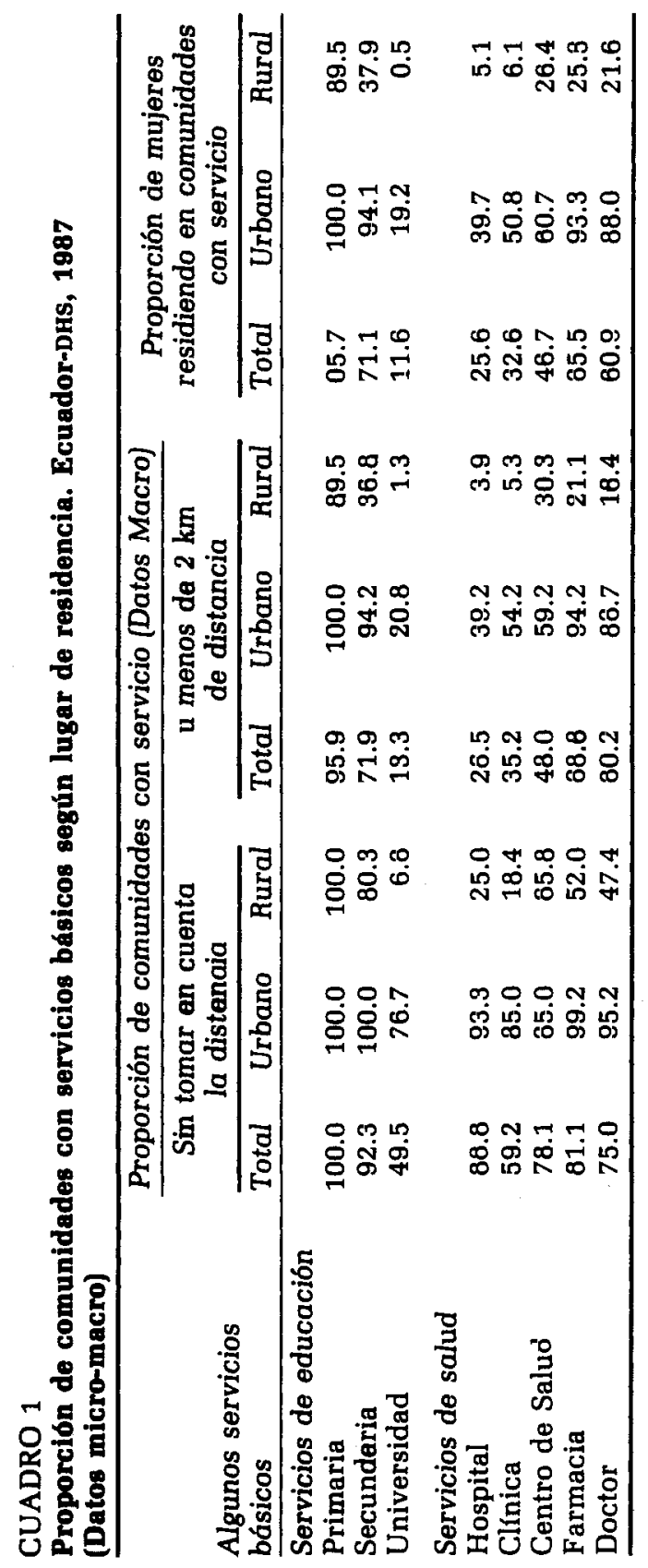


ponden a la periferia de un área urbana. Así pues, es correcta la indicación de que los conglomerados rurales cuentan con la existencia de hospitales y universidades, ya que corresponden a la zona periférica urbana.

Una característica poco común de la información de la EDS sobre disponibilidad de servicios es que se recolectó en áreas urbanas y rurales. Con el fin de sacar ventaja de esta información tan importante, se incluyeron en el análisis comunidades de ambos tipos de residencia. Cabe señalar que se podrían presentar limitaciones en la información, ya que se aplicó el mismo cuestionario para todo el país. Las preguntas son menos adecuadas para las zonas urbanas, ya que la mayoría de los servicios están disponibles en estas áreas. Hubieran sido más apropiadas preguntas sobre la densidad o la distancia entre uno y otro establecimiento.

Las instituciones y su asociación con la disminución de la fecundidad y el mejoramiento en la sobrevivencia de los hijos

Utilizando como referencia la perspectiva teórica anterior y la información de la EDS disponible, es posible hacer una estimación aproximada de la influencia de las instituciones de salud y educación sobre la mortalidad infantil y la conducta reproductiva. No se pueden medir todas las dimensiones de las instituciones de salud, como por ejemplo, los aspectos subjetivos que están involucrados en la promoción de una política específica o en la toma de una acción. Sin embargo, se podrían cuantificar los aspectos más significativos a partir del punto focal de influencia de la institución y sus agentes colaterales de salud. Esto se puede hacer si se considera la existencia y el acceso al servicio en una comunidad y la respuesta individual de la fecundidad y la mortalidad infantil a estas características contextuales. El análisis se realiza sobre la fecundidad actual y las estimaciones de mortalidad para el país en su conjunto, el lugar de residencia, urbana y rural, al momento de la encuesta sirve como un discriminador del contexto socioeconómico.

No hay una hipótesis única para el análisis. Sin embargo, considerando el marco general de acción de los servicios de salud, la hipótesis central se plantea de la siguiente manera: a través del establecimiento de normas, estrategias y mecanismos de acción, las instituciones de salud tienen un impacto positivo en las estrategias de sobrevivencia de los hijos y la fecundidad.

La variación en la intensidad del efecto de las intervenciones 
dependerá del tipo de servicio que se preste, y de su introducción en el área. Por esta razón resulta vital considerar el nivel de expansión del servicio, porque podría influenciar no solamente la localidad donde se presta, sino también las áreas que la rodean. Otro aspecto a considerar en el análisis es la naturaleza y el grado de especialización de los servicios. Para aquellos establecimientos que prestan un servicio muy especializado, uno podría reconsiderar la hipótesis central a la luz de otros factores que intervienen, tales como la selectividad de los individuos que son atraídos a estos servicios, como es el caso de los individuos migrantes a áreas donde hay servicio de universidades.

Durante la discusión, se debe tener en mente las consideraciones originales de las decisiones del gobierno $u$ otras agencias para la implantación de estos servicios. Podría suceder que se abriera un centro de salud en un lugar donde la mortalidad es más alta, y por ello, al iniciarse el servicio, la mortalidad diferencial pudiera ser más elevada que en las localidades donde no hay servicios.

A lo largo del análisis, la referencia al impacto de los servicios sobre la mortalidad infantil y la fecundidad se debe entender en un sentido aproximado. El tipo de información disponible y la complejidad de los factores que intervienen hace difícil delinear conclusiones finales sobre la causalidad, inclusive cuando se utilizan técnicas multivariadas. Los resultados se deben considerar como la primera etapa de un análisis más profundo.

En el cuadro 2 y la gráfica 1 se presentan las TMI y las TGF, para el país en su conjunto y para las áreas rurales. Como ya se mencionó, éstas se refieren a los cinco años anteriores a la fecha de la Encuesta.

Las estimaciones de la fecundidad y la mortalidad infantil de Ecuador están de acuerdo con las que han sido publicadas por la Encuesta Demográfica y de Salud y por otras fuentes (Conade, 1987; Cepar, 1988; Celade, 1990). Se estimó una tasa nacional de mortalidad infantil de 55 muertes de niños de menos de un año por cada 1000 nacidos vivos, y de 49 y 62 muertes para áreas urbanas y rurales, respectivamente. En relación con el comportamiento reproductivo, la TGF, para el país en su conjunto, se estima en 4.3 hijos. Entre el contexto urbano y el rural se presenta un amplio diferencial de dos hijos, las TGF son de 3.5 y 5.5 nacimientos respectivamente. 
Acerca de los servicios educativos y la tasa de mortalidad infantil

En relación con la existencia o falta de servicios educativos en Ecuador, se observa que hay gran disponibilidad de escuelas primarias en todo el país. En las áreas urbanas existe casi una cobertura total de este servicio. En las áreas rurales, el porcentaje de mujeres que residen en localidades donde hay escuela primaria alcanza $90 \%$. Por el contrario, los servicios de universidades para el conjunto del país son escasos (12\%) y están localizados, como ya se mencionó, exclusivamente en las áreas urbanas (véase cuadro 2).

La mortalidad infantil en relación con la educación presenta dos tendencias principales: la primera, que el acceso a los servicios de educación está asociado a una baja incidencia de muertes. La segunda muestra, tal como se estableció anteriormente, que a mayor disponibilidad de servicios educativos, la sobrevivencia de los hijos es mayor.

Para el país en su conjunto, la tasa de mortalidad infantil es más baja en comunidades donde hay servicios educativos de mayor. nivel. Así se observa que la tasa disminuye de 54.7 muertes por 1000 cuando hay disponibilidad de primaria, a $51.2 \mathrm{p} / 1000$ y $40.5 \mathrm{p} / 1000$ para áreas con escuelas secundarias y universidades, respectivamente (véanse cuadro 2 y gráfica 1).

Para el conjunto del país y las zonas rurales, las tasas de mortalidad infantil presentan diferenciales por accesibilidad a los servicios. Para las mujeres que viven en las áreas rurales, la sobrevivencia de los hijos es un poco mejor si viven en comunidades donde hay escuelas primarias (TMI de $61 \mathrm{p} / 1000$, comparada con las comunidades sin escuela: $67 \mathrm{p} / 1000$ ).

A pesar de las importantes mejoras en la sobrevivencia de los niños (y fecundidad) por la disponibilidad de los servicios educacionales o relacionados con la salud, los diferenciales urbanorural siguen presentando una brecha en la mortalidad alcanzada: los niveles son siempre más bajos para los residentes de áreas urbanas.

Acerca de los servicios de salud y la tasa de mortalidad infantil

A pesar de que la disponibilidad de servicios de educación tiene un efecto en la disminución de la mortalidad infantil, algunos de los servicios de salud tienen aún mayor relación con la sobrevivencia de los hijos.

Para el conjunto del país, la prestación de servicios de salud no es muy extensa, alrededor de $63 \%$ de las mujeres residen en una localidad donde hay una farmacia o un doctor particular; cer- 
CUADRO 2

Tasas de mortalidad infantil y tasas globales de fecundidad según disponibilidad de servicios básicos, para el total del país y las áreas rurales. Periodo de referencia 5 años. Ecuador-DHS, 1987

(Mujeres de 15-49 años)

\begin{tabular}{|c|c|c|c|c|c|c|}
\hline \multirow{2}{*}{$\begin{array}{l}\text { Servicios } \\
\text { Básicos }\end{array}$} & \multicolumn{2}{|c|}{ TMI p/1 000} & \multicolumn{2}{|c|}{ TGF } & \multicolumn{2}{|c|}{$\%$ de mujeres } \\
\hline & Total & Rural & Total & Rural & Total & Rural \\
\hline Total país & 55.4 & 61.7 & 4.3 & 5.5 & $\begin{array}{r}100.0 \\
(4713)\end{array}$ & $\begin{array}{r}40.8 \\
(1923)\end{array}$ \\
\hline
\end{tabular}

Servicios de educación

\section{Primaria}

$\begin{array}{lrrrrrr}\text { Sí } & 54.7 & 61.0 & 4.3 & 5.5 & 95.7 & 89.5 \\ \text { No } & 67.0 & 67.0 & 5.7 & 5.7 & 4.3 & 10.5 \\ \text { Dif. No-Sí } & 12.4 & 6.1 & 1.5 & 0.3 & & \\ \text { Secundaria } & & & & & & \\ \text { Sí } & 51.2 & 60.2 & 3.8 & 4.8 & 71.1 & 37.9 \\ \text { No } & 62.8 & 62.4 & 5.5 & 5.9 & 28.9 & 62.1 \\ \text { Dif. No-Sí } & 11.6 & 2.3 & 1.7 & 1.1 & & \end{array}$

Universidad

Sí

No

Dif. No-Sí

40.5

56.7

16.2

Servicios de salud Hospital

$\begin{array}{ll}\text { Sí } & 63.2 \\ \text { No } & 53.2 \\ \text { Dif. No-Sí } & -9.9\end{array}$

63.2

53.2

Clínica

\section{Sí}

No

Dif. No-Sí

Centro de Salud

Sí

No

Dif. No-Sí

$-9.9$

45.7

59.0

(-)

4.5

(-) $\quad 1.5$

11.6

0.5

88.4

99.5

Farmacia

Sí

No

Dif. No-Sí

$(-)$

$(-) \quad 4.6$

(-) 0.9

37.0

3.4

63.0

4.8

1.4

5.6

32.6

25.6

5.1

74.4

94.9

Doctor

Sí
No
Dif. No-Sí

50.8

58.6

7.8

49.0

63.7

14.7

54.5

64.0

9.4

3.9

4.7

0.9

5.4

5.6

0.1

46.7

53.3

52.3

64.3

3.7

4.6

65.5

5.6

1.9

5.8

1.2

34.5

25.2

12.0

45.4

66.5

37.2

67.4

30.2
3.6
5.4
1.8

$\begin{array}{ll}4.7 & 60.9\end{array}$

39.1

21.6

78.4
26.4

6.1

93.9

26.4 

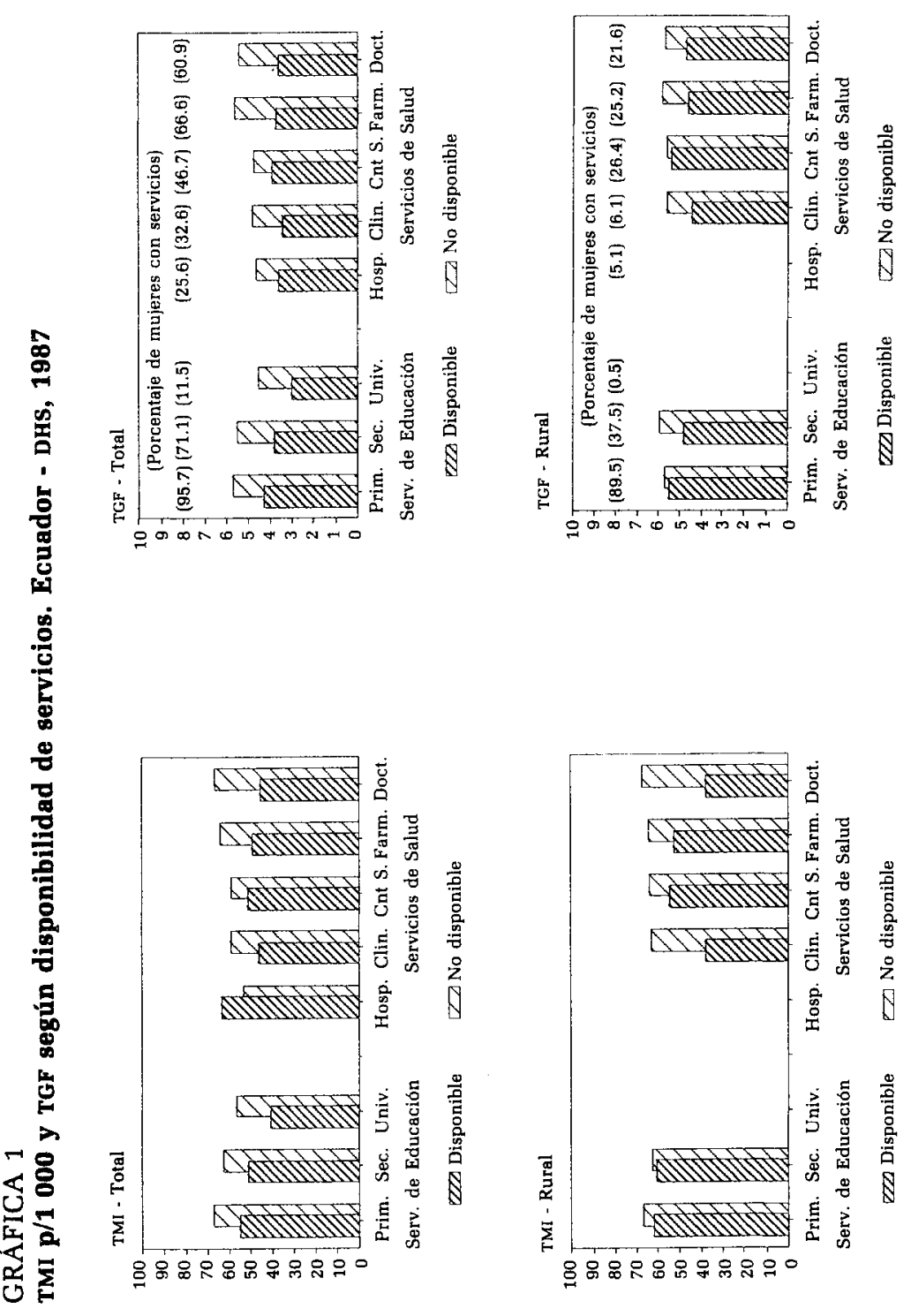
ca de la mitad de las mujeres tienen acceso a un centro de salud, y una de cada cuatro tiene acceso al hospital (véase cuadro 2).

Se puede decir que una característica de Ecuador es que en las áreas rurales hay un nivel sumamente bajo de servicios, aproximadamente sólo $25 \%$ de las mujeres tienen acceso a los cuatro establecimientos de salud disponibles en la localidad de residencia, ya que los hospitales no existen en las áreas rurales, y en el caso de las clínicas este porcentaje es aún menor, solamente $6 \%$ de las mujeres que viven en estas áreas tienen acceso al servicio.

Para la mayor parte de los establecimientos médicos se encontró una TMI más baja entre los hijos de las mujeres que residen en localidades donde hay servicios disponibles, que en aquellas donde no los hay. Esta observación es válida tanto para el país en su conjunto, como para las localidades urbanas y rurales por separado (véase gráfica 1). Para el total nacional, las áreas donde hay hospitales presentan diferenciales inversos, una tasa de 63 niños muertos por 1000 nacidos vivos, comparada con la de 53 por 1000 que se registra en áreas donde no existen hospitales. Esta diferencia no se podría atribuir a las deficiencias de la información o a sesgos introducidos en el diseño del cuestionario, ya que un examen detallado de los datos de los conglomerados y de la ubicación de los hospitales muestra, que para el caso de Ecuador, este tipo de establecimientos se construye en áreas de alta mortalidad infantil, es decir, en áreas de gran necesidad.

La más grande variación en los patrones de sobrevivencia de los hijos, para el país en su conjunto, según el tipo de servicio disponible, se observa entre las localidades que tienen acceso a un doctor particular y a las farmacias, ambos tipos de servicios pertenecen al sector privado. En las localidades rurales, los servicios que presentan los mayores diferenciales de mortalidad son los que se obtienen cuando se cuenta con la presencia del médico o la existencia de clínicas particulares ( 30 y 26 puntos de diferencia) (véase cuadro 2), es decir, establecimientos que no pertenecen al sector público.

Es interesante señalar el papel tan importante que juegan los agentes no formales de salud en la reducción de la mortalidad, en particular el de las farmacias. En éstas se pueden obtener los medicamentos y recetar a las familias, las que de otra manera no podrían obtener estos medicamentos, ya sea por no haber otros servicios médicos o por tener pocas facilidades de acceso a este tipo de establecimientos. Tal como lo veremos en la siguiente sección, esta relación también se presenta en el caso de la disminución de la fecundidad. Entre las mujeres que residen en comunidades donde no hay farmacias, la tasa de mortalidad infantil es 
$30 \%$ más alta que para las mujeres que viven en localidades donde existe este tipo de establecimiento (64 muertes infantiles por 1000 nacidos vivos, comparado con 49). La presencia de un médico también es importante para compensar o complementar la falta de instalaciones del gobierno, pero la opción de atenderse con médicos particulares tiene un efecto intrínseco de selectividad porque no todos los individuos pueden solventar el costo de este servicio.

Debido a la ausencia de hospitales en áreas rurales, las parteras pueden representar un apoyo importante a los servicios del cuidado materno-infantil y a la difusión de la información sobre planificación familiar, lo cual tendría un impacto en la sobrevivencia de los hijos y en la reducción de la fecundidad. Para el pais en general se estimó una reducción de $19 \%$ en el nivel de mortalidad infantil para los hijos de las mujeres que residen en las áreas donde hay parteras capacitadas, comparado con aquellas comunidades donde las parteras no están capacitadas -TMI de 50 y 62 por 1000 , respectivamente. Sin embargo, la proporción de parteras capacitadas todavía se mantiene baja, en particular en las zonas rurales, $78 \%$ de las familias que residen en áreas rurales disponen de servicios de parteras, de las cuales sólo $7 \%$ tiene capacitación. Estos porcentajes muestran el serio problema de la prestación de servicios en las áreas rurales, aunado a la falta de personal calificado.

Acerca de los servicios educativos y la Tasa General de Fecundidad

La disponibilidad o carencia de distintos ciclos educativos en la zona de residencia se asocia con diferenciales en la fecundidad; al comparar áreas que tienen escuelas primarias, con aquellas que cuentan con universidades, se observa una disminución en la TGF de 4.3 a 3.0, es decir, existe un diferencial de más de un hijo por mujer (véanse cuadro 2 y gráfica 1). De manera similar al caso de la mortalidad infantil, las mujeres que residen en comunidades sin servicios educativos tienen una mayor TGF que aquellas que residen en áreas donde existen estos servicios. Se estima que en las áreas donde no hay disponibilidad de servicios educativos se manifiesta un excedente de alrededor de 1.5 hijos por mujer. El nivel de fecundidad se reduce a 4.3 hijos por mujer en localidades donde hay escuelas primarias, y éste alcanza hasta 5.7 hijos en aquellas localidades sin acceso a una escuela primaria. Para el nivel secundaria, la TGF es de 3.8 en localidades que cuentan con este tipo de instituciones, y en donde no las hay la Tasa Global es de 5.5. Algo similar sucede cuando se analiza el diferencial para 
la educación universitaria; en donde existe este tipo de educación la TGF es de 3.0, y de 4.5 donde falta, tal y como se apuntó anteriormente.

Acerca de los servicios de salud y la Tasa Global de Fecundidad

De manera similar a lo que sucede con los patrones mencionados previamente, las mujeres con acceso a los servicios de salud experimentan una fecundidad más baja que aquellas en donde no están disponibles dichos servicios. Así, se estima un nivel de fecundidad de 3.4 para las mujeres que tienen acceso a las clínicas y de 4.8 para aquellas que no lo tienen; 3.9 y 4.7 cuando hay un centro de salud y un diferencial más marcado aún, de casi dos nacimientos, en el caso de las farmacias y los médicos particulares (cerca de 3.7 hijos cuando están disponibles este tipo de establecimientos y 5.5 cuando hacen falta) (véanse cuadro 2 y gráfica 1). En el análisis de los diferenciales de fecundidad, el acceso a los hospitales produce tasas de fecundidad más bajas, 3.6 hijos contra 4.6.

A pesar de que estos diferenciales aparentemente no son tan sobresalientes como aquellos que se presentaron para la TMI, en términos de porcentajes, la reducción en los niveles de fecundidad es relativamente grande. Para el total nacional, la mayor diferencia en la fecundidad se observa según el acceso a las farmacias y a los médicos. Para las áreas rurales, el centro de salud -instalaciones gubernamentales- es el servicio que tiene el menor impacto en los niveles de fecundidad (véase cuadro 2).

¿Cuáles son los rasgos sobresalientes de las respuestas demográficas de las familias a las instituciones de educación y de salud? En el caso de los servicios educativos, las respuestas no son las mismas para la TMI y para la TGF. Mientras que en las áreas rurales el acceso a las escuelas primarias es un factor importante para las diferencias en el nivel de mortalidad infantil, para la fecundidad el acceso a la escuela secundaria es el factor que más discrimina.

Con respecto a los servicios de salud existe, tanto en la sobrevivencia de los hijos como en los niveles de fecundidad, un menor impacto de las instituciones de gobierno. Para el conjunto del país, el mayor aumento en la sobrevivencia de los hijos se observa con relación a la disponibilidad de farmacias y doctores en la comunidad. Cabe señalar que estos servicios no los proporciona el gobierno y son los más abundantes en el país, posiblemente porque para su existencia se necesitan menos recursos financieros. De forma paralela las mayores modificaciones en la conducta de 
la fecundidad hacia familias más pequeñas se observa en las áreas donde existen, en primer lugar, los servicios de los médicos, y las clínicas en segundo; estas últimas requieren de un pago por parte de los usuarios. En las áreas rurales, la presencia de los servicios del sector privado tiene el mayor efecto en la sobrevivencia de los hijos y los diferenciales de la fecundidad. En el caso de la TMI es la falta de clínicas y médicos particulares, para la TGF son la existencia de farmacias y clínicas.

En Ecuador, los hallazgos de la investigación sugieren que la disponibilidad de servicios educativos y de salud genera tasas de fecundidad y de mortalidad infantil más bajas, y que el sector privado tiene un impacto relativamente más fuerte que los servicios públicos, posiblemente debido a la reducida infraestructura gubernamental del país.

Evolución de las intervenciones de salud y las modificaciones en el patrón reproductivo y la sobrevivencia de los hijos

La evolución general de estos eventos demográficos en los últimos 20 años indica una caída muy dramática, tal y como se observa en los cambios ocurridos en la TMI durante el periodo del estudio: de 107 por 1000 a 74 por 1000 , lo cual significa que ahora sobreviven 33 niños más por cada 1000 nacimientos. Por otro lado, las modificaciones en la conducta reproductiva han sido muy lentas, a pesar de que se aceleraron en la década de los ochenta, cuando en el conjunto del país se presentó un descenso de 4.7 a 3.4 hijos, en el caso de las mujeres de 15 a 35 años. Estos patrones y tendencias pueden estar ligados a la evolución histórica de las intervenciones de salud; en la presente sección se explora este concepto.

El módulo de disponibilidad de servicio de la EDS en Ecuador incluye información valiosa sobre la fecha de iniciación para los diferentes establecimientos que prestan los servicios específicos de salud y planificación familiar. Para la política del país es de gran importancia la información sobre las tendencias históricas del crecimiento del sector salud, así como de agentes de salud del sector privado. Los datos posibilitan delinear la evolución de la instauración de servicios especfficos, los que en su momento servirían como base para la evaluación del impacto de sus programas sobre las condiciones actuales de la salud y de la fecundidad. Esta sección se centra en los cambios de la fecundidad y la mortalidad infantil de zonas rurales, en relación con el acceso o no a un centro de salud, y con la prestación de servicios específicos de salud: materno-infantil, planificación familiar y rehidratación oral. 
En el cuadro 3 se presentan las estimaciones de la evolución de las TMI y las TGF para las áreas rurales, considerando la disponibilidad de los centros de salud y los servicios especfficos - programas - al momento de la ocurrencia del evento. Las gráficas 2 y 3 muestran esas tendencias, el lado izquierdo de las gráficas presenta los cambios en la TMI durante los últimos 20 años, cada estimación representa el punto medio de un periodo de 10 años. Las dos curvas describen las estimaciones de la mortalidad infantil en el periodo, conforme a la disponibilidad o no del servicio durante este lapso. De manera similar, el lado derecho de la gráfica muestra la variación en las TGF, según la disponibilidad de servicios durante los últimos 20 años. En este caso, cada estimación representa el punto medio de un periodo de cinco años. En forma adicional, se introdujo un segundo eie "Y" en el lado derecho de las gráficas, el cual denota el porcentaje de mujeres con acceso a este servicio de salud o programa.

\section{Acerca de los centros de salud}

Las tendencias de la mortalidad muestran una clara relación con las facilidades de servicios de salud. Los centros de salud y los establecimientos de gobierno indican estar localizados originalmente en áreas rurales de alta mortalidad con más de 130 muertes infantiles por 1000 nacimientos (véanse cuadro 3 y gráfica 2). Durante el periodo en estudio, la mortalidad descendió a 45 por 1000 , lo que significa una reducción de más de la mitad del nivel de la década anterior. Entre las mujeres que viven en áreas sin servicios, también se registra una disminución en la mortalidad infantil, pero con una velocidad de descenso menor, de 106 por 1000 a 79 por 1000 . Esto no resulta sorprendente, debido al posible impacto de los diversos elementos tales como el efecto sinergético de la disponibilidad de servicios de salud, la aplicación de una tecnologia médica moderna tal como las campañas de vacunación, y otros factores socioeconómicos del desarrollo, tales como la introducción de agua potable y obras de saneamiento. Sin embargo, para el conjunto del pafs, el acceso a los centros de salud resultó en una ganancia adicional de sobrevivencia de cerca de $\mathbf{3 0 0} 000$ niños durante el periodo 1967-1986, lo que significó una reducción de 78 puntos en la TMI.

En el caso de la fecundidad, los cambios no son tan impresionantes como aquellos que se observan para la mortalidad infantil. El impacto que causan en la conducta de los individuos el acceso a los centros de salud o a los programas y las variaciones en las 


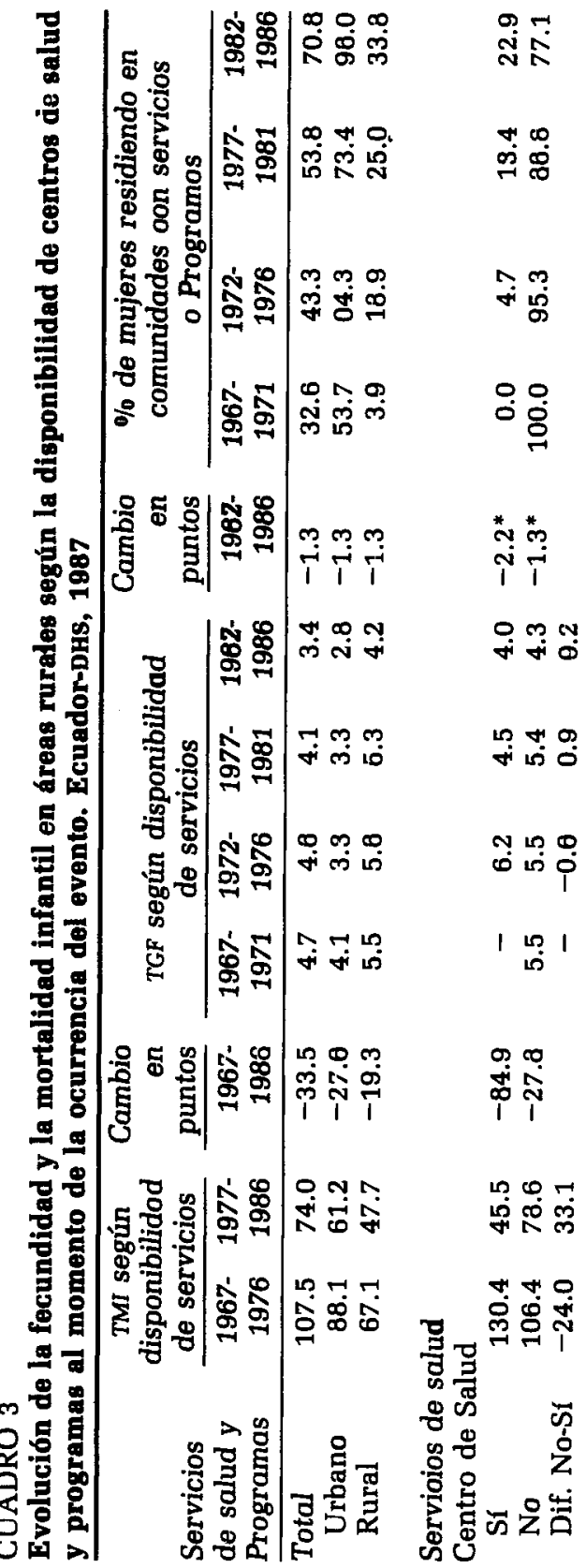


REDUCCIÓN DE LA FECUNDIDAD Y LA MORTALIDAD INFANTIL 399

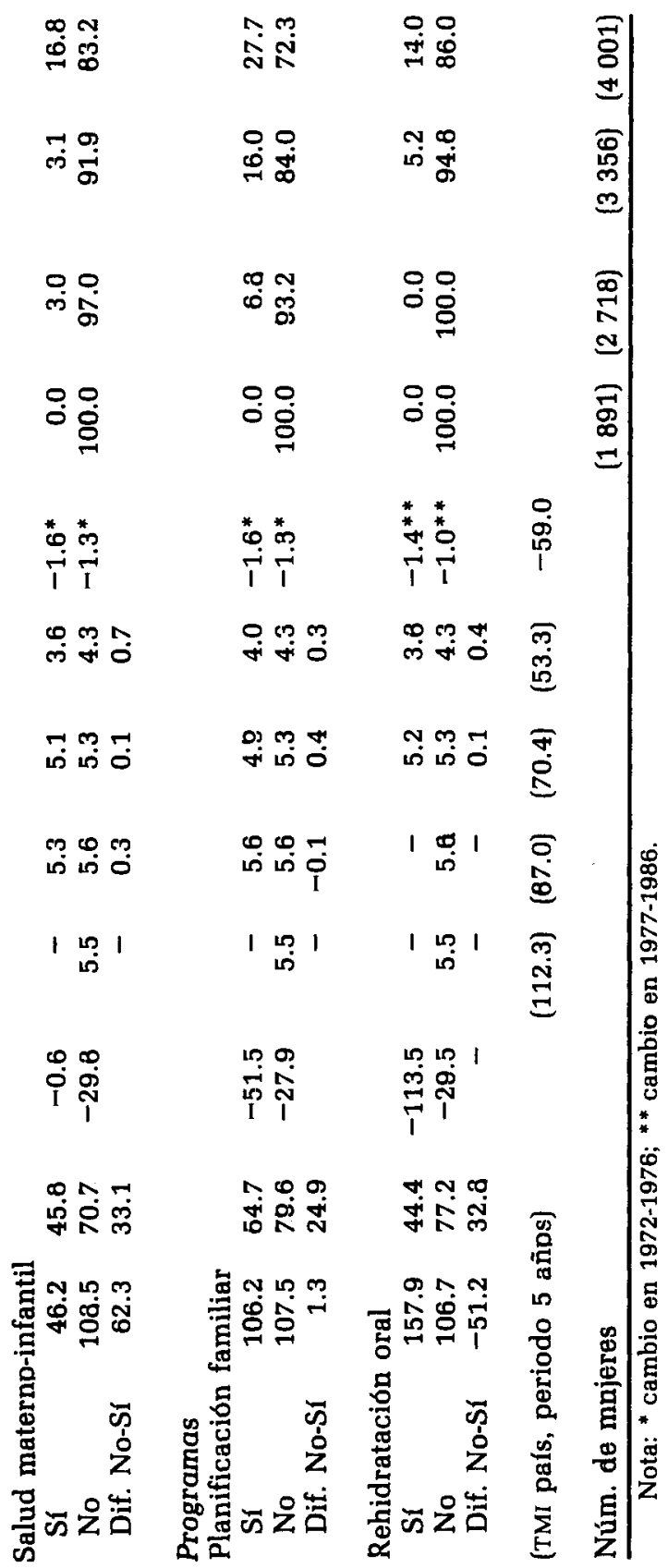




\section{GRÁFICA 2}

TMI y TGF según disponibilidad de Centro de Salud en el momento de la ocurrencia del evento. Ecuador - DHs, 1987. Área rural

\section{Centro de salud}
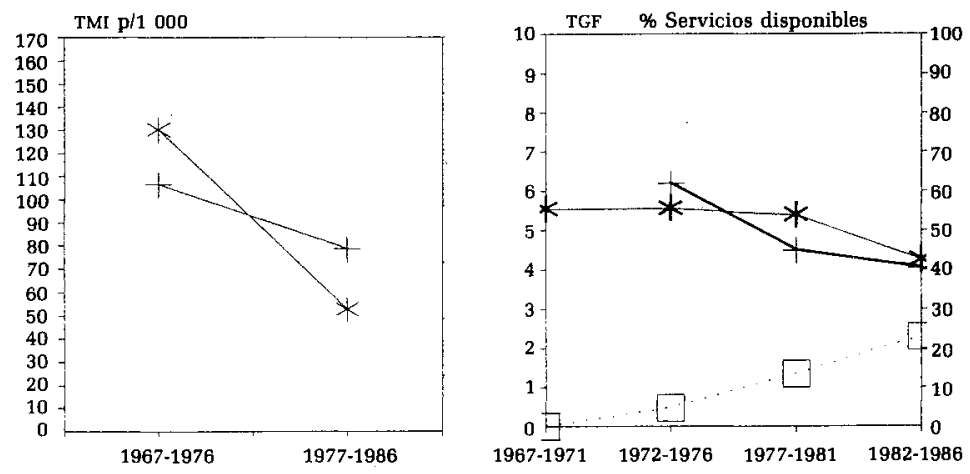

* Sí $\quad+$ No

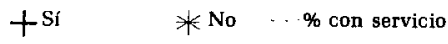

condiciones socioeconómicas de la comunidad, no necesariamente pueden manifestarse de inmediato en modificaciones de la conducta reproductiva. La adopción de nuevas normas hacia la reducción del tamaño de la familia puede surgir como resultado de un contacto más prolongado de las condicionantes externas. Para el periodo en cuestión, el nivel de la fecundidad permaneció más o menos constante, y los principales cambios ocurrieron en los últimos cinco años. La reducción en la tasa, durante los últimos 20 años, es muy modesta, primeramente porque la fecundidad no era extremadamente alta al inicio del periodo, y segundo porque la transición hacia el descenso empezó muy recientemente. Para el área rural hay una disminución de la fecundidad de 1.3 hijos en un lapso de 20 años, empezando con 5.5 hijos al inicio del periodo y llegando a 4.2 hijos para la fecha más reciente (véanse cuadro 3 y gráfica 2).

En el pasado, la existencia de centros de salud estaba asociada con grandes diferenciales en el comportamiento reproductivo, es decir que se ubicaron, en su inicio, en áreas con altos niveles de fecundidad. Sin embargo, en la actualidad sólo hay una modesta variación en los niveles de fecundidad según el acceso o no a los centros de salud. Durante el periodo analizado, las mujeres resi- 
dentes en áreas con este tipo de instalaciones experimentaron una reducción en la fecundidad de 2 hijos.

\section{Acerca de los programas}

Debido a la naturaleza de los programas que se consideraron en el análisis: cuidado materno-infantil, planificación familiar y rehidratación oral, se espera que algunos puedan afectar de manera más significativa a la sobrevivencia de los niños y otros al comportamiento reproductivo. Pero es posible que la respuesta a los programas de salud pudiera tener un efecto combinado sobre la fecundidad y la mortalidad infantil. Además, pudiera suceder que las estimaciones muestren efectos indirectos debido a la interrelación de estos dos eventos demográficos. La respuesta de la fecundidad y la mortalidad no son independientes una de otra, ya que las acciones e intervenciones dirigidas a modificar el comportamiento reproductivo también podrían tener impacto en la salud y en la condición de sobrevivencia de niños y adultos. Un aspecto adicional que debe ser considerado es que la conducta de los individuos puede derivar hacia una respuesta cultural distinta a la que prevalecía en torno al concepto de salud, tal sería el caso de algunas mujeres que reaccionan favorablemente al acceso tanto de los programas como de los servicios de salud que inciden en la sobrevivencia de los hijos así como a los programas de planificación familiar dirigidos al espaciamiento de los hijos y a la reducción de la fecundidad.

Al considerar los programas de salud, salud materno-infantil, rehidratación oral y planificación familiar, las tasas de mortalidad infantil muestran otra vez modificaciones impresionantes. Las áreas con acceso al programa de salud del cuidado materno-infantil tienen una mortalidad relativamente baja, cerca de 45 por 1000 , la cual permanece constante durante el periodo analizado. La existencia de un programa de rehidratación oral crea una marcada diferencia en la respuesta de la mortalidad. Al principio de los años setenta los servicios de rehidratación oral llegaron a las áreas en donde se registraba una mortalidad infantil extremadamente alta: 158 por 1000 . En la actualidad, las mujeres que viven en localidades donde hay este programa muestran una TMI de sólo 44 por 1000 . También se observa una tendencia descendente en áreas donde no hay acceso a la rehidratación oral, pero con ganancias en la sobrevivencia de los hijos no tan fuertes como entre los hijos de las mujeres que tienen acceso a este programa. Se presentan patrones similares en torno a los centros de salud y a los otros programas (véase gráfica 3 ). 
GRAFICA 3

TMI y TGF según disponibilidad de programas en el momento de la ocurrencia del evento. Ecuador - DH8, 1987. Área rural
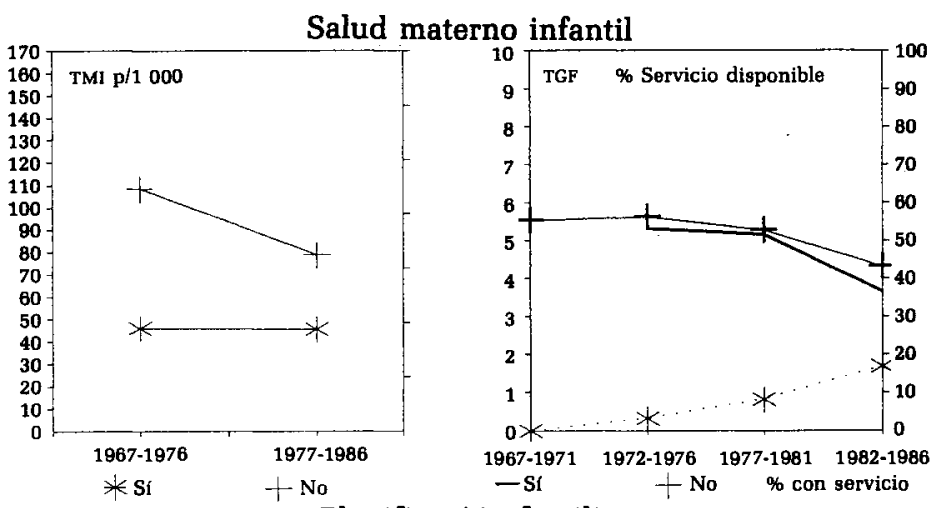

Planificación familiar
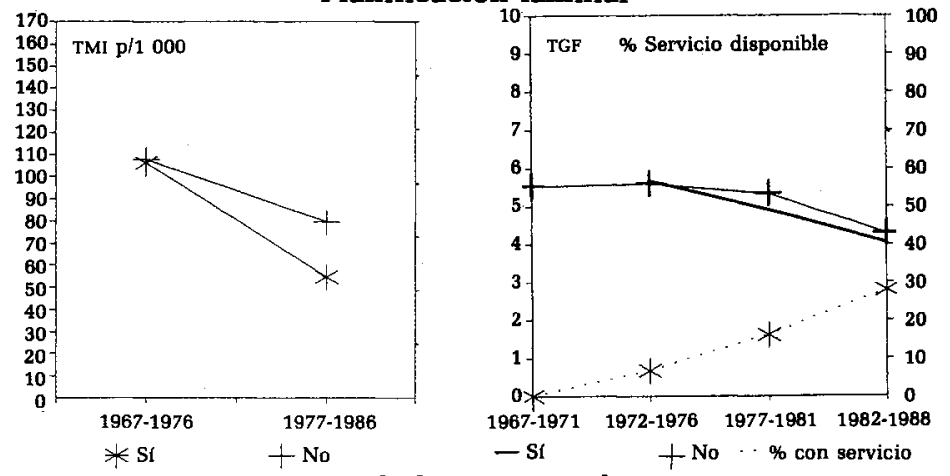

Rehidratación oral
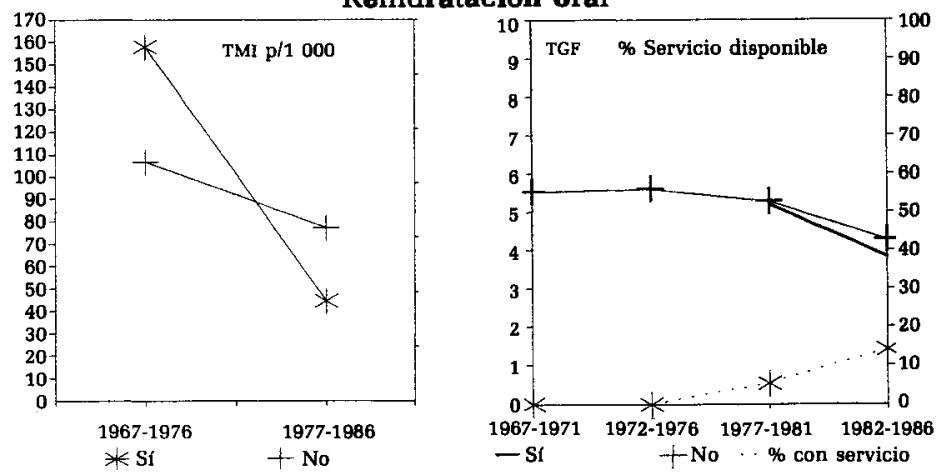
Durante las primeras etapas de la introducción de los programas de planificación familiar no se encontró diferencia en la TMI para las mujeres que viven en áreas donde se tiene o es inexistente el acceso a los programas. Para periodos más recientes se presenta un mejoramiento en la sobrevivencia de los hijos; los niveles de la mortalidad infantil se vieron modificados fuertemente, ya que de 106 muertes infantiles por 1000 nacimientos se redujo a 55 por 1000 en áreas con acceso a los programas de planificación familiar, que es un cambio más significativo si se compara con las localidades donde no existen servicios de planificación familiar (véanse cuadro 3 y gráfica 3 ).

Los patrones de fecundidad no muestran grandes variaciones y, como descolla en el caso del análisis anterior, no fue sino hasta recientemente que el comportamiento reproductivo ha empezado a cambiar en las áreas rurales. La única modificación que llama la atención es la respuesta un poco más marcada hacia el programa de salud materno-infantil. Las mujeres en áreas rurales con acceso a él experimentan una gran disminución en su comportamiento reproductivo en comparación con aquellas que residen en localidades que carecen de éste. En este caso, pudiera ser que los servicios del programa de salud materno-infantil incorporaran los elementos de planificación familiar, lo cual podría explicar el gran impacto que tiene el acceso a este programa en la conducta reproductiva de las mujeres.

Comparado con los otros programas analizados, el de planificación familiar es el que cubre una mayor proporción de mujeres de áreas rurales: $30 \%$, y el acceso a los otros programas es muy bajo (véanse cuadro 3 y gráfica 3).

Los resultados muestran claramente que la existencia y el acceso a los programas específicos están fuertemente asociados con los niveles más bajos de mortalidad infantil y de la fecundidad. Se puede asumir con seguridad que la expansión de estos servicios y programas seguirá contribuyendo a la reducción de los niveles de la mortalidad infantil y la fecundidad.

\section{Conclusiones}

La creciente influencia y el control de las instituciones de salud sobre los aspectos que en el pasado se pensó que estaban influidos únicamente por las acciones individuales, hace muy significativo el papel de las instituciones de salud -particularmente en lo que se refiere a la puesta en práctica de los servicios y programasy de sus agentes complementarios, como es el sector privado. 
La falta de estudios que consideran las variables de la comunidad en el análisis de la fecundidad y la mortalidad nos impulsó a realizar este trabajo sobre el caso particular de Ecuador. El presente estudio puede servir como ejemplo de la magnitud del impacto de la institución salud en estos eventos demográficos.

Los hallazgos indican que la disponibilidad de los servicios juega un papel de suma importancia en el comportamiento del individuo con respecto a la fecundidad y a la mortalidad infantil. Al considerar al país como un todo, las farmacias y los médicos, es decir, los agentes de salud no gubernamentales, parecen tener el mayor impacto en los niveles actuales de fecundidad y mortalidad infantil. En el contexto rural surge una respuesta distinta a la del área urbana, en cuanto a la manera en que las instituciones influyen en el comportamiento demográfico, lo que se explica en parte por el bajo nivel de desarrollo alcanzado en este contexto y la escasa prestación de servicios.

La inspección simultánea de la evolución de los servicios rurales y las tendencias de la fecundidad y la sobrevivencia de los hijos muestra resultados interesantes que son un estímulo, tanto desde la perspectiva de la recolección de la información, como por la posibilidad de medir el impacto de las instituciones de salud. El análisis indica que hay una respuesta coherente y compatible en los niveles de la fecundidad y la mortalidad infantil en cuanto al acceso a los centros de salud, pero es aún más fuerte en relación con los programas específicos. El acceso a la rehidratación oral aumentó la sobrevivencia infantil en mayor medida que el acceso a los otros programas de salud, ya sean públicos o privados. En contraposición a lo que se hubiera esperado, la fecundidad no muestra una gran respuesta como resultado de la disponibilidad de programas de planificación familiar. Los mayores diferenciales en el comportamiento reproductivo se observan cuando hay acceso a los servicios del cuidado materno-infantil.

El módulo de disponibilidad de servicio de la EDS ha mostrado que es relevante para el estudio de la relación entre las esferas micro y macro. Sin embargo, resulta esencial la reformulación del diseño de dicho cuestionario con el objeto de eliminar una gran cantidad de preguntas que no captan la información para la que fueron diseñadas.

Este documento es un primer paso exploratorio para resaltar la importancia de la asociación entre la disponibilidad de servicios y el comportamiento de la fecundidad y la mortalidad infantil. 


\section{Bibliografía}

Casterline, H. (ed.) (1985), The Collection and Analysis of Community Data, wFs Seminar on "Collection and Analysis of Data on Community and Institutional Factors", ISI/WFS, 20-23 de junio, 1982.

Camarena, R. M. (1991), Instituciones sociales y reproducción, México.

Celade (1990), "Latin America: Population Projection, 1950-2025", en Demographic Bulletin, año XXII, núm. 45, Santiago de Chile, enero.

Cepar (1988), Ecuador. Encuesta Demogrăfica y de Salud Familiar 1987, Centro de Estudios de Población y Paternidad Responsable, Instituto Nacional de Investigaciones Nutricionales y Médico Sociales, Institute for Resource Development/Westinghouse, abril.

Conade-Unfpa (1987), Población y cambios sociales, Quito.

Entwisle, B. (1989), "Measuring Components of Family Planning Program Effort", en Demography, vol. 26, núm. 1, febrero.

Hermalin, A. (1986), "The Multi-Level Approach: Theory and Concepts", en The Methodology of Measuring the Impact of Family Planning Programmes on Fertility, Population Studies núm. 66, Addendum, Manual IX, United Nations.

INEC (1985), IV Censo de Población, 1982. Resultados definitivos, Resumen Nacional, Ecuador-Noviembre de 1982, Quito.

Loyola, M. A. y M. C. Quintero (1982), Instituções e reprodução. Estudo da atuaçăo das instituções sociais no processo da reprodução humana, São Paulo, Brasil, Estudos de População, CEBRAP.

McNicoll, G. (1980), "Institutional determinants of fertility change", en Population and Development Review, vol. 6, núm. 3.

(1982), "Institutional determinants of fertility change", en C. Hohn y R. Mackensen (ed.), Determinants of Fertility Trends: Theories Re-Examined, Lieja, Bélgica, IUSsP, Ordina Editions.

y M. Cain (1990), "Rural Development and Population. Institutions and Policy", en Population and Development Review, suplemento, vol. 15, 1989.

Potter, J., O. Mojarro y L. Núñez (1987), "The Influence of Health Care on Contraceptive Acceptance in Rural Mexico", en Studies in Family Planning, vol. 18, núm. 3.

Tsui, O. y L. H. Ochoa (1989), "Service Proximity as a Determinant of Contraceptive Behaviour: Evidence from Cross-National Studies of Survey Data", documento presentado en el Seminario "The role of family planning programs as a fertility determinant", IUSSP, Túnez, 26-30 de junio. 
